\title{
Remogliflozin Etabonate
}

National Cancer Institute

\section{Source}

National Cancer Institute. Remogliflozin Etabonate. NCI Thesaurus. Code C91023.

An orally available prodrug of remogliflozin, a benzylpyrazole glucoside-based inhibitor of renal sodium-glucose co-transporter subtype 2 (SGLT2) with antihyperglycemic activity. Upon administration and absorption, the inactive prodrug is converted to its active form remog liflozin and acts selectively on the sodium-glucose co-transporter subtype 2 (SGLT2), 